\title{
Long-term outcome after high tibial osteotomy
}

\author{
Michaela Gstöttner · Florian Pedross •
}

Michael Liebensteiner · Christian Bach

Published online: 15 January 2008

(C) Springer-Verlag 2008

Erratum to: Arch Orthop Trauma Surg (2008) 128:111-115 DOI 10.1007/s00402-007-0438-0

The authors' first names and family names were transposed.

The correct list of authors is:

Michaela Gstöttner · Florian Pedross ·

Michael Liebensteiner · Christian Bach

The online version of the original article can be found under doi:10.1007/s00402-007-0438-0.

M. Gstöttner $(\square) \cdot$ M. Liebensteiner $\cdot$ C. Bach Department of Orthopedic Surgery,

Medical University Innsbruck, Anichstraße 35,

6020 Innsbruck, Tirol, Austria

e-mail: Michaela.gstoettner@uklibk.ac.at

F. Pedross

Department of Medical Statistics,

Informatics and Health Economics,

Medical University Innsbruck, Anichstraße 35,

Innsbruck, Tirol, Austria 